\title{
A Tentative Design of a Future Internet Networking Domain Landscape
}

\author{
Marc Pallot, Brigitte Trousse, and Bernard Senach \\ INRIA Sophia Antipolis - Méditerranée, AxIS Project-team, France
}

\begin{abstract}
The Future Internet (FI) will dramatically broaden both the spectrum of available information and the user's possible contexts and situations. This will lead to the vital need of a more efficient use of the Internet resources for the benefit of all. While the Internet has already delivered huge economic and social benefits over its short lifespan, there must be a realignment of how Internet research and investments are made and value is captured for enabling a continuous growth. The increase of available online contents and networking complexity require the exploration, experimentation and evaluation of new performance optimisation approaches for delivering different types of contents to users within different contexts and situations. Several network research areas, such as peer-to-peer, autonomous, cognitive and ad hoc networking, have already demonstrated how to improve network performance and user experience.

Interestingly, there are various Internet-networking research areas and corresponding technologies that were investigated, experimented and progressively deployed, while others emerged more recently. However, there are still open questions such as visualising the conceptual evolution and articulating the various FI networking and computing research areas and identifying appropriate concepts populating such a FI domain landscape. This paper presents a tentative FI domain landscape populated by Internet computing and networking research areas.
\end{abstract}

Keywords: Future Internet, Internet-Networking, Domain-Landscape, NetworkComputing, Internet-Routing.

\section{Introduction}

The Internet has progressively become a ubiquitous environment for globally communicating and disseminating information. There is a limitless amount of available online resources and tools to share information and develop a better understanding on whatever topics. With the recent advent of user created content, thanks to the web 2.0 social approach, there has been a tremendous expansion in the number of web pages created every day for exposing and sharing societal issues such as environmental monitoring, energy efficiency, food and drug security as well as human well-being. Tools like photo/video sharing, mash-ups, tagging, wikis and collaborative virtual worlds enable new ways for the society to explore and understand past present and future challenges. The Future Internet (FI) will 
dramatically broaden both the spectrum of available information and the user's possible contexts and situations. This will lead to the vital need of a more efficient use of the Internet resources for the benefit of all. While the Internet has already delivered huge economic and social benefits over its short lifespan, there must be a realignment of how Internet research and investments are made and value is captured for enabling a continuous growth.

\section{Future Internet Networking Domain Landscaping}

\subsection{The Future Internet}

Pirolli and colleagues [1] argue that an extensive research is required for building upon currently used Internet media and tools to foster wider user participation to tackle US national priorities through technology-mediated social participation. Mobile and Internet technologies have converged into ubiquitous social connectivity. Pirolli and colleagues report that in spring 2010, 40\% of adults (aged 30 and over), $72 \%$ of young adults and $73 \%$ of teens use social network web-sites and that time spent on Facebook increased by more than $500 \%$. They also mention that many vibrant communities have emerged on the Web such as Wikipedia having more than 12 million registered users and more than 3 million content pages and in February 2010, Twitter users generated 35 million tweets per day.

The term "Future Internet" (FI) represents worldwide research activities for reinventing the Internet with better performance, reliability, scalability, security and privacy while keeping its key neutral principle as constantly recommended by Tim Beemer's-Lee, the famous inventor of the Web. As shown in the FI networking domain landscape (see Figure 1), there is a great diversity of research streams and related topics for designing alternatives of the Internet networking of tomorrow. For example, the Internet of Things (IoT) is considered as a major research and innovation stream leading to create plenty of service opportunities in interconnecting physical and virtual worlds with a huge amount of electronic devices (e.g. sensors, actuators) distributed in houses, vehicles, streets, buildings and many other public environments (e.g. airports, train, metro and bus stations, social spaces). Hence, a massive amount of data will be flowing over the Internet that should not decrease the overall service performance and user satisfaction.

The movement towards the Future Internet is based on the belief that the current Internet has reached his limits. Tselentis [2] states: "The current Internet has been founded on a basic architectural premise, that is: a simple network service can be used as a universal means to interconnect intelligent end systems. This simple premise has allowed the Internet to reach an impressive scale in terms of inter-connected devices. However, while the scale has not yet reached its limits, the growth of functionality and the growth of size have both slowed down. It is now a common belief that the current Internet would reach soon both its architectural capability limits and its capacity limits."

The FI represents the evolving need for infrastructures at the level of innovation infrastructure (networks of collaboration, experimental facilities, research and test centres etc), and broadband Internet infrastructure (networks, services). Recently, 
several testbeds were initiated, such as PlanetLab [3], TEFIS, BonFIRE and SensLAB [2]. TEFIS supports the Future Internet of Services Research by offering a single access point to different testing and experimental facilities for communities of software and business developers to test, experiment, and collaboratively elaborate knowledge [4], [5]. The main goal of the BonFIRE project is to design, build and operate a multi-site Cloud prototype FIRE facility to support research across applications, services and systems at all stages of the R\&D lifecycle, targeting the services research community on Future Internet. The purpose of the SensLAB project is to deploy a very large scale open wireless sensor network platform, in order to provide an accurate and efficient scientific tool to help in the design and development of real large-scale sensor network applications. SensLAB has been instrumental in detecting overlapping communities in complex networks [6].

Challenging issues arise from the study of dynamic networks like the measurement, analysis and modelling of social interactions, capturing physical proximity and social interaction by means of a wireless network. A concrete case study exhibited the deployment of a wireless sensor network applied to the measurement of Health Care Workers' exposure to tuberculosis infected patients in a service unit of the Bichat-Claude Bernard hospital in Paris, France [7]. As described above through different testbed projects, the Future Internet is the "provider" of future Internet infrastructure and applications. Obviously, the Future Internet will be the key driver of technological support for services and products to be explored, experimented and evaluated.

\subsection{Towards a Tentative Future Internet Networking Research Domain Landscape}

While working on the development of a Living Lab research domain landscape on methods for involving users in $\mathrm{R} \& \mathrm{D}$ [8] and discussing about the scientific program of the FIA event, it came to our mind that it could be useful to prepare a map as a tentative FI landscape populated by Internet computing and networking research areas. Furthermore, several INRIA research teams are involved in FIRE Testbed projects, namely: PlanetLab, OneLab, TEFIS, SensLAB, and BonFIRE whose scientific leaders were interviewed during the development of this tentative FI landscape.

We believe that the proposed landscape of FI networking research domain could provide a faster and broader understanding of the different research streams and related topics. Several dimensions were used for landscaping the FI networking research domain:

- Evolution approaches: from incremental (evolution) design to Clean Slate redesign or radical evolution from where emerge new generation networks;

- Internet routing: from the basic data packet delivery towards more sophisticated content distribution and retrieval capacities (content Centric Networking);

- Network Types: from wired communication (cable or optical networks) to wireless communication networks (wireless Internet, wireless sensors networks);

- Evolution trends: from a traditional computer network towards an autonomic and convergent network that become the computing network. 
A number of concepts representing various research areas were selected for populating the domain landscape. The selection of concepts was carried out in parsing a large set of published papers in order to identify prominent terms that correspond to research publication streams, which were validated during the interviews. The following six categories were identified:

- Network Computing: Pervasive Computing, Ubiquitous Computing, Grid Computing, and Cloud Computing;

- Internet Routing: Peer-to-Peer Network, Ad-hoc Network, Content Centric Networking, Self-adaptive Network, Resilient Network, Fault tolerant Network, Autonomic Network and Cognitive Network;

- Network Type: Wireless Sensor Network, Optical Network, Wireless Internet;

- Network Security: Virtual-private Network, Internet Security;

- Network Assessment: Quality of Services, Quality of Experience;

- Network (IP) Globalisation: Next Generation Network, IP Multimedia Subsystem, Internet of Things, Network Convergence.

Two categories are clearly identical to two of the above mentioned dimensions, namely: "Internet Routing" and "Network Type". A third category, "Network Computing", quite overlaps with the dimension named "Evolution trends".

As for the category "Network Computing", it is worth to note that the concept of Pervasive Computing, often mentioned as the 'disappearing computing', and Ubiquitous Computing, rather evoked as 'computing is everywhere' are often used synonymously especially in the Ambiance Intelligence area. In the same vein, the concept of Grid computing, known as a cluster of networked computers, and Cloud Computing, computing as a service or storage as a service, are quite closely related from the perspective of shared resources. Regarding the category of Network Globalisation, all the concepts are related to the convergence towards 'all $\mathrm{IP}^{1}$, strategy and to the concepts of the Network Computing category as well as the Internet Routing category. The Network Security and Network Assessment categories have more transversal concepts that need to be considered at the earlier stage of the FI design.

For each research stream, a Google scholar search over three different time periods was carried out as a publication metric intended to show their respective growth or decline. All selected concepts, considered as research areas, are individually described in the Table 4 Appendix at the following URL ${ }^{2}$. The respective levels of publication for each concept are provided in the table below (see Table 1) showing the publication values for the three respective time periods, and sorted by ascending value of the column 2006-2011.

\footnotetext{
${ }^{1}$ Internet Protocol.

${ }^{2}$ http: / / www. mosaic-network.org/pub/bscw. cgi/0/69097
} 
Table 1. Foreseen Concepts Belonging to the FI Networking Research Domain Landscape

\begin{tabular}{|l|r|r|r|}
\hline \multicolumn{1}{|c|}{$\begin{array}{c}\text { Concepts } \\
\text { (research areas) }\end{array}$} & \multicolumn{3}{|c|}{ Number of Papers } \\
\hline Content-centric Networking & 81 & 1 & -1999 \\
\hline Self-adaptive network & 88 & 70 & 0 \\
\hline Resilient Network & 424 & 179 & 26 \\
\hline Fault tolerant network & 544 & 326 & 57 \\
\hline Autonomic Network & 715 & 151 & 390 \\
\hline Cognitive Network & 1370 & 377 & 25 \\
\hline Network Convergence & 1760 & 988 & 273 \\
\hline Quality of Experience & 2230 & 672 & 566 \\
\hline Internet of Things & 2400 & 117 & 91 \\
\hline Optical Networking & 2500 & 2450 & 8 \\
\hline IP Multimedia Subsystem & 2800 & 604 & 234 \\
\hline Next Generation Network & 4030 & 1650 & 3 \\
\hline Peer-to-Peer Network & 6780 & 3630 & 206 \\
\hline Quality of Services & 6970 & 5300 & 122 \\
\hline Internet Security & 7030 & 6130 & 1050 \\
\hline Wireless Sensor Network & 7320 & 1140 & 1390 \\
\hline Semantic Web Services & 7990 & 2390 & 16 \\
\hline Virtual Private Network & 8100 & 6930 & 11 \\
\hline Cloud Computing & 10200 & 127 & 844 \\
\hline Wireless Internet & 12400 & 8440 & 144 \\
\hline Ad hoc Network & 12500 & 7160 & 332 \\
\hline Grid Computing & 15100 & 7870 & 291 \\
\hline Ubiquitous Computing & 15300 & 12200 & 75 \\
\hline Pervasive Computing & 15600 & 8970 & 1230 \\
\hline & & & 129 \\
\hline
\end{tabular}

The bar-graph below (see Figure 1) shows the growth in terms of published papers for the respective selected concepts across the three different time-periods. The highest level of publication belongs to the concepts of the category "Network Computing" and Ad hoc Network as well as Wireless Internet. However, the growth rate of Cloud Computing looks so impressive that it is quite easy to predict it as the next big thing on the Future Internet. Not surprisingly, among other concepts having an impressive growth rate are Wireless Sensor Network and Internet of Things. The lowest level of published papers appears to be related to more emerging concepts of the Internet Routing category, such as Content Centric Networking, Self-adaptive Network, Resilient Network, Fault-tolerant Network and Cognitive Network.

The growth rate of Virtual Private Network is impressively decelerating in the last time-period while it had an impressive growth rate in the middle time-period. The same evidence appears to apply on Internet Security and Quality of Services. The situation is even worst in terms of growth rate for Optical Networking, which seems to have reached its maximum amount of annual publication. 


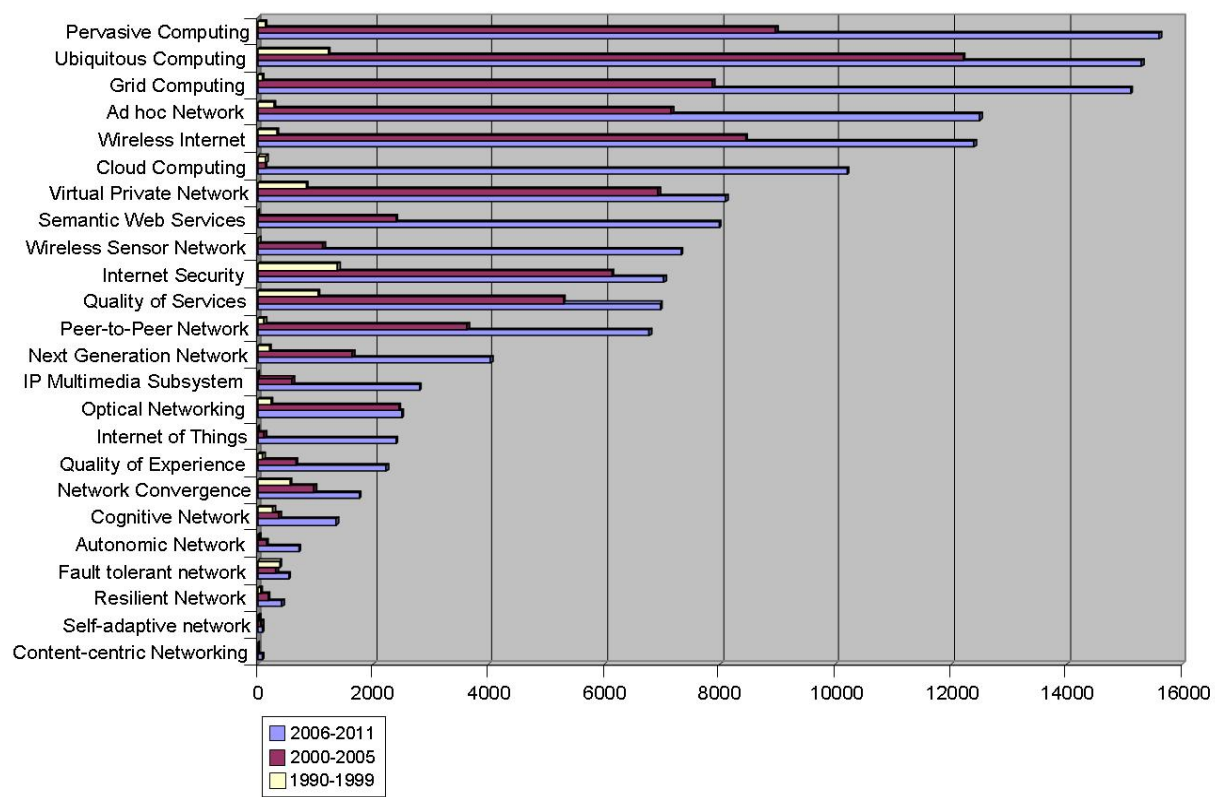

Fig. 1. Publication Metric for FI Networking Research Concepts

The landscape is divided twice. First of all, it is divided in two spaces: a top space and a bottom space that respectively address the wired and wireless Internet. Secondly, it is divided in a right hand located space corresponding to the more traditional "Computer Network" and in a left hand space representing the more recent "Network Computing".

A tentative design of the Future Internet networking research domain landscape for three successive time periods appears below (see Figures 2, 3 and 4) where each concept, presumed research area, appears as a bubble whose size is proportional to the overall amount of publication in the corresponding time-period. The various concepts and their allocated bubbles populate the landscape according to the four different dimensions.

\section{FI Networking Domain Landscape for the time period 1990-1999}

The FI networking research domain landscape for the time period 1990-1999 appears in Figure 2 where the concept of "Network Computer" forms a big island on the left hand side due to its publication level of 16400 published papers. The opposite island, about 5 times smaller, is constituted by the concept of "Network Computing" with 3340 published papers in the same period. The concept of "Ubiquitous Computing" belonged to "Network Computing" with a publication level of 1230 published papers during this period of time. The biggest bubbles represent the most published aspects at that time such as "Optical Networking", "Virtual Private Network", "Ad Hoc Network", "Quality of Service”, "Internet Security" and "Wireless Internet”. Smaller 
bubbles represent less published aspects such as "Peer-to-Peer Network", "Network Convergence", "Cognitive Network" and "Fault Tolerant Network". More surprisingly, the idea of "Next Generation Network" existed already with 250 published papers by end of year 1999 .

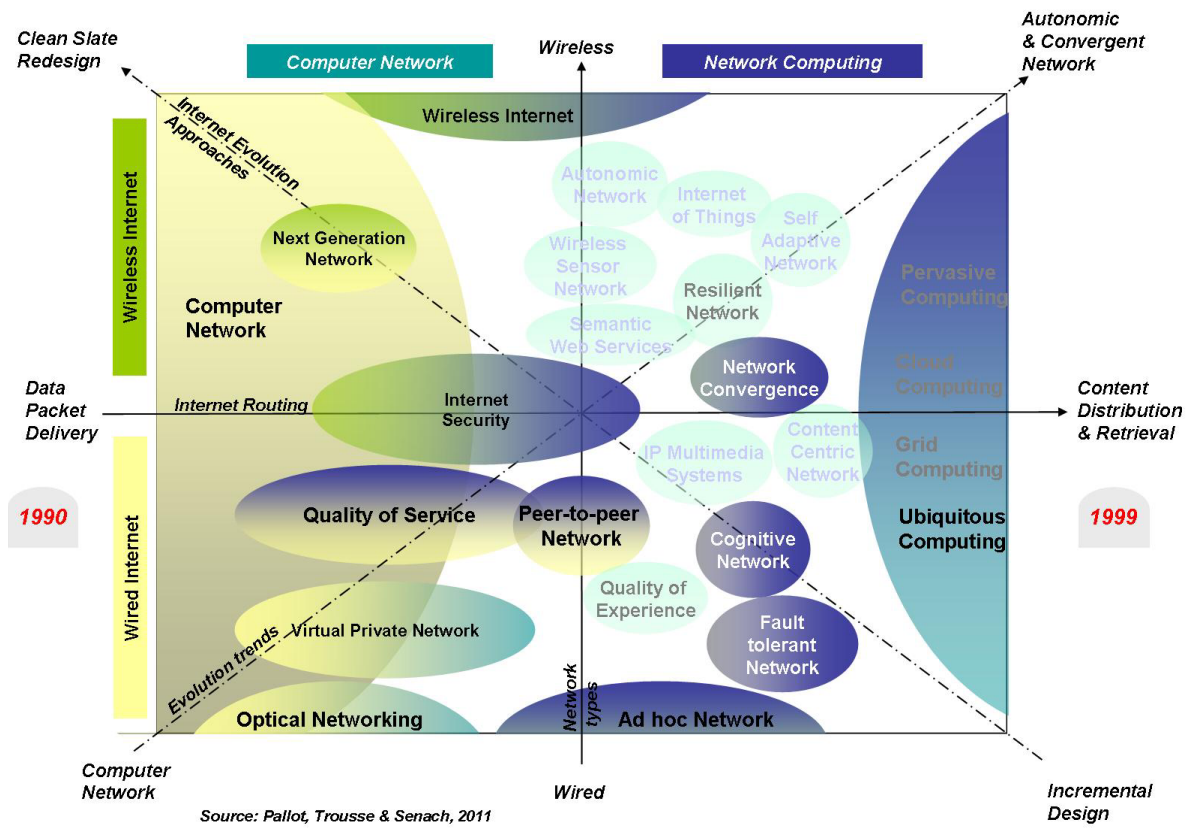

Fig. 2. FI Research Domain Landscape 1990-1999

On the vertical dimension from wired to wireless Internet, the island in the bottom area is constituted of "Optical Networking" while the island in the top area is based on "Wireless Internet". The islands on the right and left hand spaces as well as the islands in the bottom and top spaces are supposed to generate a certain gravity attracting other concepts through the other dimensions of internet routing, evolution approach and autonomic \& convergent network.

All low brightness small bubbles represents emerging aspects with very few published papers such as "IP Multimedia System" counting 3 published papers and "Internet of Things" with 8 published papers. The only concept that was not emerging by year 1999 is represented by "Content Centric Networking", which scored 0 published papers, has a very low brightness level in the figure.

\section{FI Networking Domain Landscape for the time period 2000-2005}

The FI networking research domain landscape for the time period 2000-2005 appears in Figure 3 where the concept of "Network Computer" forms a bigger island due to an increased publication level of 19200 published papers. The opposite island, constituted by the concept of "Network Computing", is in this period only 4 times smaller due to a double amount of 5860 published papers in the same period. 
Interestingly, "Wireless Internet" has considerable grew up to 12400 published papers as well as "Optical Networking", hence, both generate much more gravity and attraction. For example the concept of "Wireless Sensor Network" has turned from an emerging bubble into a real one with 1140 published papers. However, in this same period of time, "Internet of Things" has only scored 117 published papers.

Other concepts have turned from emerging concepts to confirmed ones, such as "Semantic Web Services", "Quality of Experience", "Internet of Things", Cognitive Network", "Autonomic Network", "Self Adaptive Network" and "Resilient Network" as well as "Cloud Computing" that respectively scored in Google Scholar 2390, 672, $117,377,151,70,179$ and 127 published papers.

Similarly to the previous period of time, "Content Centric Networking" has still a very low brightness level in the figure because it scored only 1 published paper,

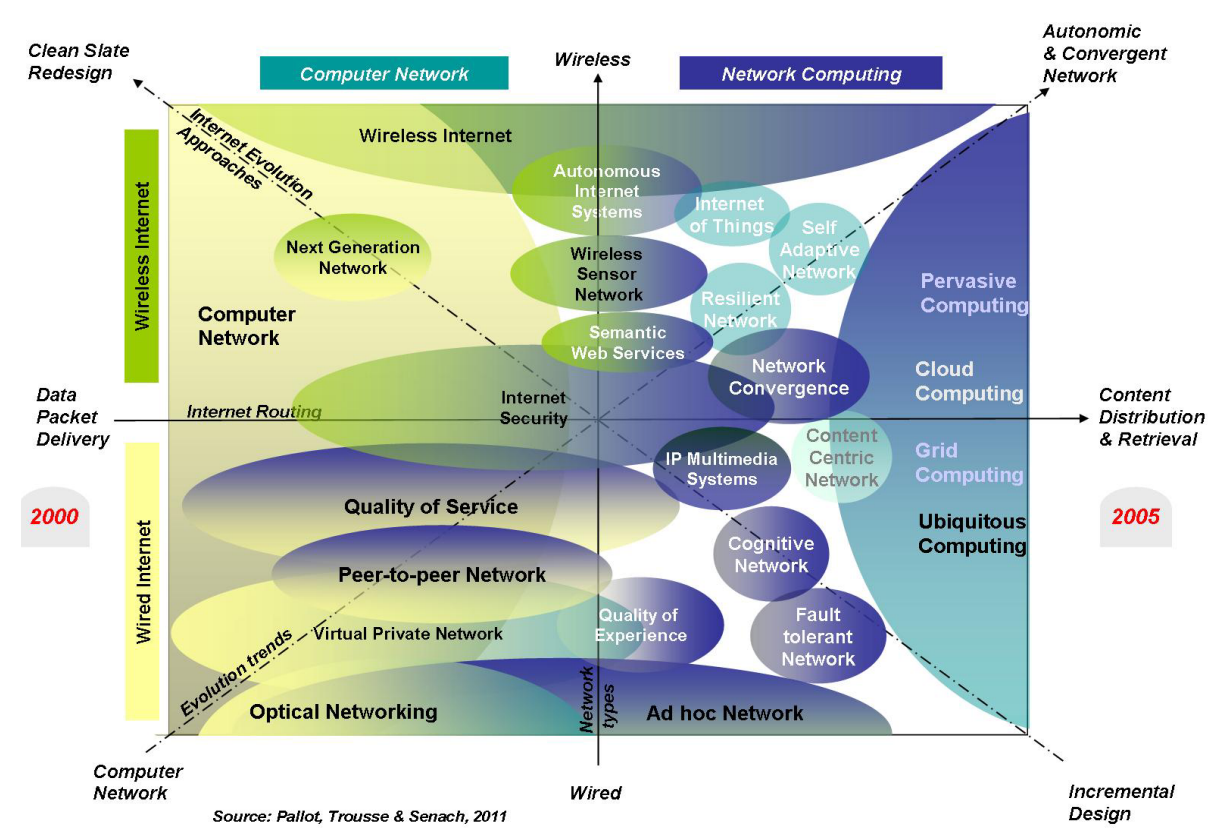

Fig. 3. FI Research Domain Landscape 2000-2005

\section{FI Networking Domain Landscape for the time period 2006-2011}

The FI networking research domain landscape for the time period 2006-2011 appears in Figure 4 where the concept of "Network Computer" starts to decrease with a publication level of 18100 published papers. The opposite island, "Network Computing", increases with 6960 published papers. This might be highlighting the current transition from network computer towards network computing. The "Contentcentric Networking" concept emerges in this period with 81 published papers.

The most impressive progression comes from the concept of "Cloud Computing" that exponentially moves from 127 published paper in the previous period (2000-2005) up to 10200 in this period (almost factor 100). The concept of "Internet 
of Things" moves in the same way but with a less exponential (factor 20) progression from 117 to 2400. There are other concepts that make a good progression in this period, such as "Autonomic Network", "Wireless Sensor Network", "Cognitive Network" and "Quality of Experience". Finally, the concept of "Next Generation Network" makes also a significant progression in moving from 1650 to 4030 published paper in the period 2006-2011.

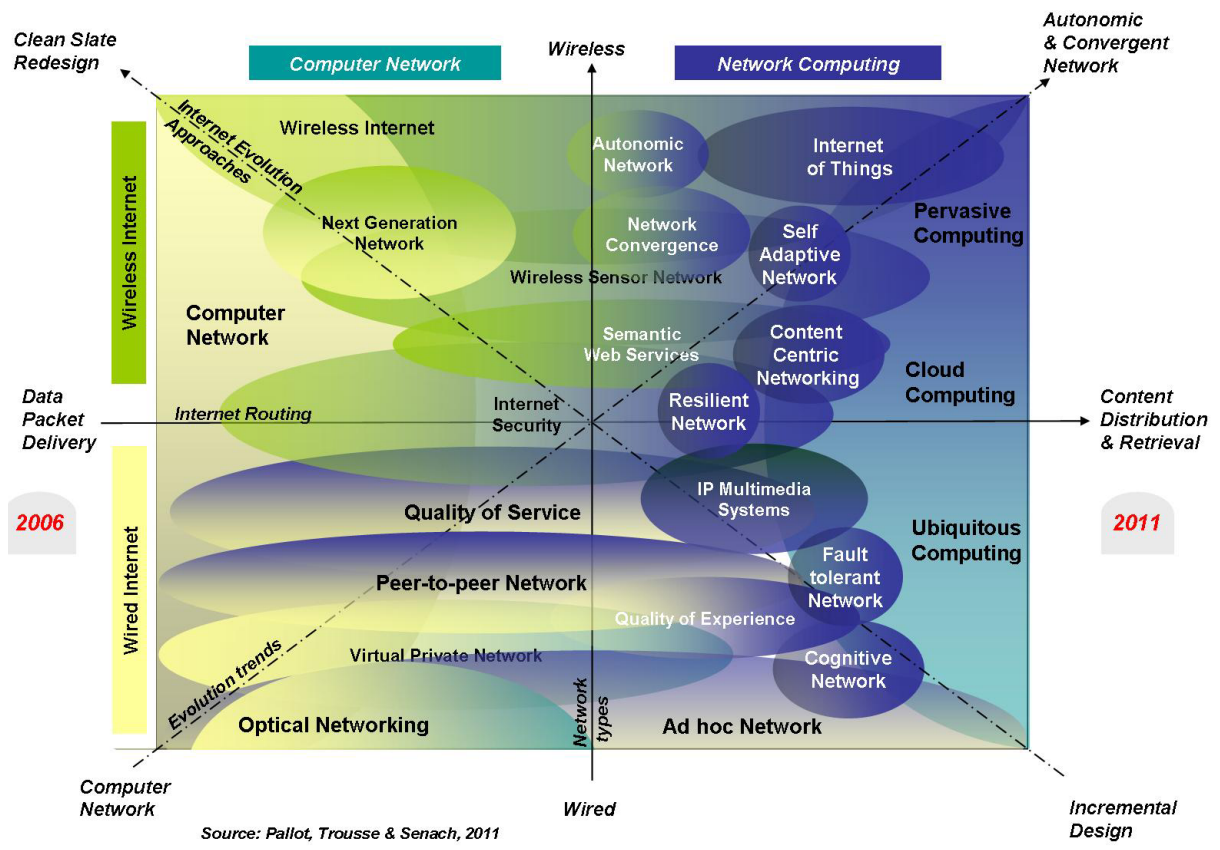

Fig. 4. FI Research Domain Landscape 2006-2011

As for the concepts having a stable publication level over the two period 2000-2005 and 2006-2011, "Optical Networking" and "Internet Security" have a very small increase of respectively $2 \%$ and $13 \%$. The concept of "Quality of Services" is also on the way to reach a stable plateau kind of situation with $20 \%$ progression compare to the previous progression (5 times) from 1990-1999 to 2000-2005 publication levels.

\section{Evolution of Interest in FI Networking Research Areas}

A ranking from 1 to 24 was computed for each period based on the amount of published papers. The evolution of interest in FI research areas reflected by the publication weight of the respective FI concepts appears in Table 2, which highlights the concept or research area 'Pervasive Computing' as the most popular for the time period 2006-2011 and 'Content-Centric Networking' as the less popular. 
Table 2. Ranking of Concepts along the different time-periods

\begin{tabular}{|l|c|c|c|}
\hline \multirow{2}{*}{\multicolumn{1}{|c|}{ Concepts }} & \multicolumn{3}{|c|}{ Rank } \\
\cline { 2 - 4 } & $2006-2011$ & $2000-2005$ & $1990-1999$ \\
\hline Content-centric Networking & 24 & 24 & 24 \\
\hline Self-adaptive network & 23 & 23 & 18 \\
\hline Resilient Network & 22 & 19 & 17 \\
\hline Fault tolerant network & 21 & 18 & 6 \\
\hline Autonomic Network & 20 & 20 & 19 \\
\hline Cognitive Network & 19 & 17 & 9 \\
\hline Network Convergence & 18 & 14 & 5 \\
\hline Quality of Experience & 17 & 15 & 15 \\
\hline Internet of Things & 16 & 22 & 22 \\
\hline Optical Networking & 15 & 10 & 10 \\
\hline IP Multimedia Subsystem & 14 & 16 & 23 \\
\hline Next Generation Network & 13 & 12 & 11 \\
\hline Peer-to-Peer Network & 12 & 9 & 14 \\
\hline Quality of Services & 11 & 11 & 3 \\
\hline Internet Security & 10 & 7 & 1 \\
\hline Wireless Sensor Network & 9 & 13 & 20 \\
\hline Semantic Web Services & 8 & 8 & 21 \\
\hline Virtual Private Network & 7 & 6 & 4 \\
\hline Cloud Computing & 6 & 21 & 12 \\
\hline Wireless Internet & 5 & 3 & 7 \\
\hline Ad hoc Network & 3 & 5 & 8 \\
\hline Grid Computing & 2 & 4 & 16 \\
\hline Ubiquitous Computing & 1 & 2 & 2 \\
\hline Pervasive Computing & & & 13 \\
\hline
\end{tabular}

It shows as well that several FI research areas (concepts) while they were part of the most popular in the time period 1990-1999, became the less popular in the time period 2006-2011, such as 'Fault Tolerant' (from rank 6 to rank 21), 'Network Convergence' (from rank 5 to rank 18), 'Cognitive Network' (from rank 9 to rank 19), 'Quality of Services' (from rank 3 to rank 11) and finally 'Internet Security' (from rank 1 to rank 10).

Others remain in the most popular, such as 'Ubiquitous Computing' (from rank 2 to rank 2), 'Ad hoc Network' (from rank 8 to rank 4), and 'Wireless Internet' (from rank 7 to rank 5). Finally, FI research areas that were the less popular in the time period 1990-1999, became the most popular in the time period 2006-2011, such as 'Grid Computing' (from rank 16 to rank 3), 'Pervasive Computing' (from rank 13 to rank 1), 'Cloud Computing' (from rank 12 to rank 6), 'Semantic Web Services' (from rank 21 to rank 8) and 'Wireless Sensor Network' (from rank 20 to rank 9). 
Table 3. Ranking Evolution of FI Concepts in Two Time-Periods

\begin{tabular}{|c|c|c|}
\hline \multirow[b]{2}{*}{ Concepts } & \multicolumn{2}{|c|}{ Ranking Evolution } \\
\hline & 2011-2006 & 1990-1999 \\
\hline Content-centric Networking & 0 & 0 \\
\hline Self-adaptive network & 0 & -5 \\
\hline Resilient Network & -3 & -2 \\
\hline Fault tolerant network & -3 & -12 \\
\hline Autonomic Network & 0 & -1 \\
\hline Cognitive Network & -2 & -8 \\
\hline Network Convergence & -4 & -9 \\
\hline Quality of Experience & -2 & 0 \\
\hline Internet of Things & 6 & 0 \\
\hline Optical Networking & -5 & 0 \\
\hline IP Multimedia Subsystem & 2 & 7 \\
\hline Next Generation Network & -1 & -1 \\
\hline Peer-to-Peer Network & -3 & 5 \\
\hline Quality of Services & 0 & -8 \\
\hline Internet Security & -3 & -6 \\
\hline Wireless Sensor Network & 4 & 7 \\
\hline Semantic Web Services & 0 & 13 \\
\hline Virtual Private Network & -1 & -2 \\
\hline Cloud Computing & 15 & -9 \\
\hline Wireless Internet & -2 & 4 \\
\hline Ad hoc Network & 1 & 3 \\
\hline Grid Computing & 1 & 12 \\
\hline Ubiquitous Computing & -1 & 1 \\
\hline Pervasive Computing & 1 & 11 \\
\hline
\end{tabular}

Interestingly, several FI networking research areas have a non homogeneous ranking in the middle time period, such as 'Cloud Computing' with a transition from rank 12 (1990-1999) to 21 (2000-2005) and finally 6 (2006-2011). Most of the other research areas display a progression or regression. The most constant is the research area 'Ubiquitous Computing' with the ranking 2-1-2 and 'Next Generation Network' with ranking 11-12-13 as well as 'Autonomic Network' with ranking 19-20-20.

Another way of looking at the ranking of FI networking research areas consists in considering the number of lost/gained positions between 1 and 24 within the time periods of 2006-2011 and 1990-1999 compared to the middle one 2000-2005. The following table highlights the FI networking research areas with the highest gain, such as 'Cloud computing' with a considerable gain of 15 position and 'Internet of Things' with a less impressive gain of 6 position (see Table 3). During the previous period (1990-1999), the winning three were Semantic Web Services, Grid Computing and Pervasive Computing. 


\section{$4 \quad$ Remarks and Conclusions}

After digging into all these figures, one might be thinking that Cloud Computing and Internet of Things research publication streams will continue to exponentially grow due to the current interest in developing innovative services based on the 'open data' strategy developed by cities that deploy more and more sensors in their urban areas. Surprisingly, Content Centric Networking appears to be quite flat in terms of publication stream while current networks mainly move content objects through not optimum host-to-host conversations. Is it due to a lack of research projects in the $\mathrm{CCN}$ area? Or is it simply due to the anticipated deployment difficulties?

The lack of domain landscape on FI networking research appears to be a potential topic of interest for researchers for the elaboration of the FI roadmap and related networking research challenges for the next 10 years. It would help to reach a broader understanding of the location and articulation of the various networking concepts.

Exploring the research domain landscape of FI and identifying related networking concepts in digging into a large amount of published papers was a demanding but useful task. The design of the FI landscape with concept bubbles inhabiting the various territories reveals to be more fascinating. During this work, a territory of "computing" emerged as a necessary bubble linking more recent concepts.

Developing a landscape on a wider FI domain could be useful for the research community in order to identify and locate FI related concepts within dimensions showing possible directions of progress. However, it would logically require the participation and contribution of the whole FI research community. We hope that this first tentative and issued FI landscape of networking concepts will motivate enough other researchers for contributing to its future development.

Acknowledgements. This work was performed within the FIREBALL ICT project and partly funded by the European Commission. The authors wish to acknowledge the European Commission and project partners for their support. We also wish to acknowledge our gratitude and appreciation to INRIA scientific leaders of project teams involved in FI related projects, namely: Denis Caromel and Franca Perrina (TEFIS), Walid Dabbous (PlanetLab and OneLab), Eric Fleury (SensLAB), and David Margery (BonFIRE), for their active contribution.

Open Access. This article is distributed under the terms of the Creative Commons Attribution Noncommercial License which permits any noncommercial use, distribution, and reproduction in any medium, provided the original author(s) and source are credited.

\section{References}

1. Pirolli, P., Preece, J., Shneiderman, B.: Cyberinfrastructure for Social Action on National Priorities. IEEE Computer Journal, special Issue on Technology-Mediated Social Participation (November 2010)

2. Tselentis, G., Galis, A., Gavras, A., Krco, S., Lotz, V., Simperl, E., Stiller, B., Zahariadis, T.: Towards the Future Internet - Emerging Trends from European Research (2010), http: / / www . booksonline. iospress.nl/Content/View.aspx?piid=16 465 (retrieved) 
3. Burin des Rosiers, C., Chelius, G., Fleury, E., Fraboulet, A., Gallais, A., Mitton, N., Noël, T.: SensLAB Very Large Scale Open Wireless Sensor Network Testbed. In: Korakis, T., Li, H., Tran-Gia, P., Park, H.-S. (eds.) TridentCom 2011. LNICST, vol. 90, pp. 239-254. Springer, Heidelberg (2012)

4. Kaafar, M., Mathy, A., Barakat, L., Salamatian, C., Turletti, K., Dabbous, T.: Securing Internet Coordinate Embedding Systems. In: Proceedings of ACM SIGCOMM 2007, Kyoto, Japan (August 2007)

5. Schaffers, H., Santoro, R., Sällström, A., Pallot, M., Trousse, B., Hernandez-Munoz, J.M.: Integrating Living Labs with Future Internet Experimental Platforms for Co-creating Services within Smart Cities. In: Proceedings of the 17th International Conference on Concurrent Enterprising, ICE 2011 Innovating Products and Services for Collaborative Networks, Aachen, Germany (June 2011)

6. Leguay, J., Sallstrom, A., Pickering, B., Boniface, M., Gracia, A., Perrina, F., Giammatteo, G., Roberto, J., Campowsky, K.: Testbed Facilities for Multi-faceted Testing throughout the Service Development Lifecycle. In: ServiceWave 2011, Poznan (October 2011)

7. Friggeri, A., Chelius, G., Fleury, E., Fraboulet, A., Mentré, F., Lucet, J.-C.: Reconstructing Social Interactions Using an unreliable Wireless Sensor Network. Computer Communications 34(5), 609-618 (2011a)

8. Friggeri, A., Chelius, G., Fleury, E.: Egomunities, Exploring Socially Cohesive Personbased Communities, $\mathrm{N}^{\circ}$ RR-7535 (2011) [inria-00565336 - version 2] (2011b)

9. Pallot, M., Trousse, B., Senach, B., Scapin, D.: Living Lab Research Landscape: From User Centred Design and User Experience towards User Cocreation. In: Proceedings of the Living Lab Summer School, Cité des Sciences, Paris (August 2010) 